\section{Assessment of thyroid-specific quality of life in patients with benign symptomatic thyroid nodules treated with radiofrequency or ethanol ablation: a prospective multicenter study}

So Yeong Jeong ${ }^{1}$, Eun Ju Ha², Jung Hwan Baek', Tae Yong Kim³ ${ }^{3}$ Yu-Mi Lee ${ }^{4}$, Jeong Hyun Lee', Jeonghun Lee ${ }^{5}$

'Department of Radiology and Research Institute of Radiology, Asan Medical Center, University of Ulsan College of Medicine, Seoul; ${ }^{2}$ Department of Radiology, Ajou University School of Medicine, Suwon; ${ }^{3}$ Department of Internal Medicine, ${ }^{4}$ Division of Endocrine Surgery, Department of Surgery, Asan Medical Center, University of Ulsan College of Medicine, Seoul; ${ }^{5}$ Department of Surgery, Ajou University School of Medicine, Suwon, Korea

Purpose: Radiofrequency ablation (RFA) and ethanol ablation (EA) are effective and safe for benign symptomatic thyroid nodules (BSTNs). However, relatively little is known about the effects of these procedures on patients' quality of life (QoL). This prospective, multicenter study evaluated the effects of RFA and EA on changes in thyroid-specific QoL in patients with BSTNs and assessed the volume reduction and safety of these procedures.

Methods: Eighty-six consecutive patients with 86 BSTNs were prospectively included from two medical centers. RFA was performed for 55 BSTNs with solidity $\geq 50 \%$ and EA was performed for 31 BSTNs with solidity $<50 \%$. QoL was evaluated using an 11-scale, multiple-choice thyroidspecific QoL questionnaire. Nodule characteristics and QoL were evaluated at diagnosis and 1, 6, and 12 months after treatment. Overall QoL was rated from 0 (good) to 4 (poor).

Results: The mean longest size and volume of the index nodule were $4.2 \pm 1.5 \mathrm{~cm}$ and $21.6 \pm 22.1$ $\mathrm{mL}$, respectively. Patients received 1.1 treatments on average (range, 1 to 2). Significant posttreatment volume reductions were noted; however, the EA group showed a higher volume reduction than the RFA group at $1(78.7 \%-16.1 \%$ vs. $49.1 \%-15.8 \%), 6(86.3 \%-21.7 \%$ vs. $73.0 \%-14.5 \%)$, and $12(90.9 \%-14.9 \%$ vs. $80.3 \%-12.4 \%)$ months. The score for each scale of the QoL questionnaire improved significantly during follow-up (all $\mathrm{P}<0.001$ ). Overall QoL improved significantly, from $1.7 \pm 0.9$ at diagnosis to $0.6 \pm 0.7$ at the 12 -month follow-up $(\mathrm{P}<0.001)$. There were no major complications.

Conclusion: Both RFA and EA are safe and effective in reducing nodule volume and improving thyroid-specific QoL in patients with BSTNs.

Keywords: Radiofrequency ablation; Ethanol; Quality of life; Thyroid nodule

Key points: Radiofrequency ablation (RFA) and ethanol ablation (EA) significantly improved all thyroid-specific quality of life scales and overall quality of life in patients with benign symptomatic thyroid nodules. Patients with cystic nodule treated with EA had higher volume reduction than patients with solid nodule treated with RFA at 1-, 6-, and 12-months.
ULTRA

SONO

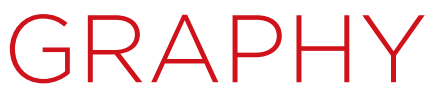

ORIGINAL ARTICLE

https://doi.org/10.14366/usg.21003 pISSN: 2288-5919 - elSSN: 2288-5943 Ultrasonography 2022;41:204-211

Received: January 4, 2021

Revised: June 12, 2021

Accepted: June 29, 2021

Correspondence to: Jung Hwan Baek, MD, PhD, Department of Radiology and Research Institute of Radiology, Asan Medical Center, University of Ulsan College of Medicine, 88 Olympic-ro 43-gil, Songpa-gu, Seoul 05505, Korea

Tel. +82-2-3010-4348

Fax. $+82-2-476-4719$

E-mail: radbaek@naver.com

This is an Open Access article distributed under the terms of the Creative Commons Attribution NonCommercial License (http://creativecommons.org/ licenses/by-nc/4.0/) which permits unrestricted noncommercial use, distribution, and reproduction in any medium, provided the original work is properly cited.

Copyright (? 2022 Korean Society of Ultrasound in Medicine (KSUM)

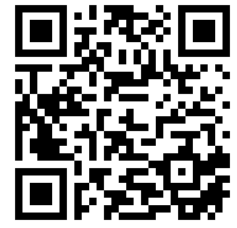

How to cite this article:

Jeong SY, Ha EJ, Baek JW, Kim TY, Lee YM, Lee $\mathrm{JH}$, et al. Assessment of thyroid-specific quality of life in patients with benign symptomatic thyroid nodules treated with radiofrequency or ethanol ablation: a prospective multicenter study. Ultrasonography. 2022 Jan;41(1):204211.

e-ultrasonography.org 


\section{Introduction}

Thyroid nodules are common clinical problems. Although most thyroid nodules are benign and asymptomatic, some are symptomatic and require treatment. Surgery has long been the treatment of choice for patients with benign symptomatic thyroid nodules (BSTNs); however, since the introduction of the minimally invasive treatments for the management of BSTNs, current international practice guidelines [1-4] recommend minimally invasive methods (e.g., thermal or chemical ablation) as an alternative to surgery, as these methods can minimize the drawbacks of surgery and improve patients' quality of life (QoL).

Among the minimally invasive methods currently employed to treat BSTNs, radiofrequency ablation (RFA) and ethanol ablation (EA) have been used widely to reduce nodule volume and improve nodule-related symptoms and cosmetic problems $[5,6]$. Although many previous studies, including randomized clinical trials and meta-analyses, have confirmed the effectiveness and safety of these procedures for the management of BSTNs [3,7-9], relatively little is known about the effects of these procedures on patient QoL. Regarding QoL after ablation treatment, several studies [1012] have reported positive outcomes, whereas another study by Oddo et al. [13] showed the opposite results. In addition to causing compressive symptoms and cosmetic problems, thyroid nodules can affect other various aspects of daily life, including physical, social, and emotional well-being. Thus, whether ablation treatments improve thyroid-specific QoL should be verified in order to weigh the advantages and disadvantages appropriately.

Therefore, the primary purpose of this prospective, multicenter study was to evaluate the effects of RFA and EA on changes in thyroid-specific QoL in patients with BSTNs, and the secondary purpose was to evaluate the volume reduction ratio (VRR) and safety of these procedures.

\section{Materials and Methods}

\section{Compliance with Ethical Standards}

The protocol of this study was approved by the Ethics Committees of the Institutional Review Boards of the two participating centers (Asan Medical Center and Ajou University Hospital, institutional review board number: 2015-0608). Written informed consent was obtained from all patients before they underwent RFA or EA.

\section{Participation}

Between January 2015 and April 2016, 95 BSTNs from 95 consecutive patients were identified from the two participating centers (Fig. 1). After excluding six patients who declined to participate, 89 patients were treated with RFA or EA. After treatment, three patients were lost to follow-up before 6 months and were also excluded. A final sample of 86 patients with 86 BSTNs was included in this prospective study. RFA was performed for 55 BSTNs with a solid component $\geq 50 \%$ and EA was performed for 31 BSTNs with a solid component $<50 \%$.

The eligibility criteria for inclusion were as follows: (1) patients who reported symptoms or cosmetic problems due to a single thyroid nodule or a predominant thyroid nodule in a goiter, (2) benign cytology confirmed through the evaluation of at least two separate ultrasound (US)-guided fine-needle aspiration samples or core needle biopsies $[14,15]$, (3) normal thyroid function tests, and (4) patients who were considering undergoing EA or RFA. The exclusion criteria were as follows: (1) patients who were younger than 20 years, (2) thyroid nodules with suspicious sonographic

95 Nodules from 95 consecutive patient between January 2015 and April 2016

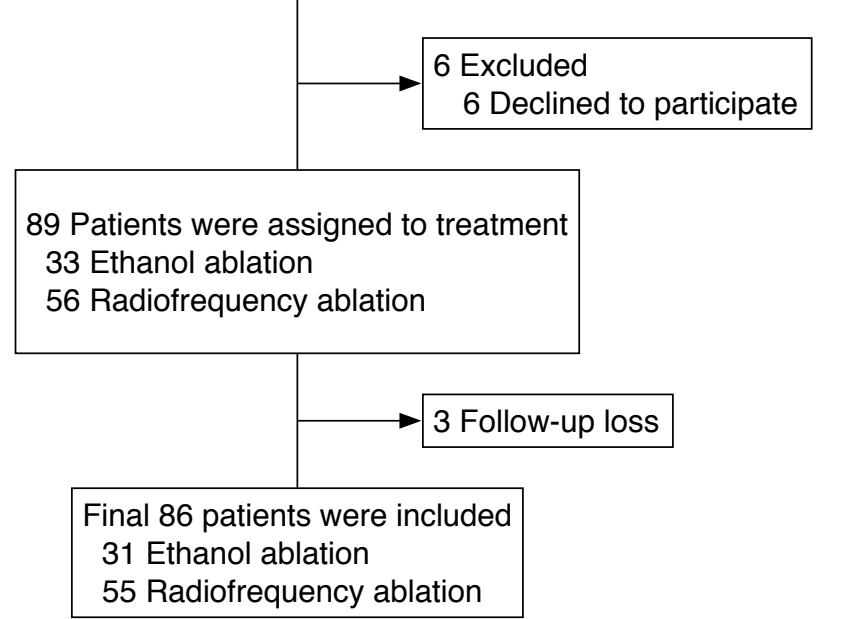

Fig. 1. Flow chart of this study. 
features, (3) abnormal thyroid function tests, and (4) pregnancy.

\section{Preprocedural Assessment}

All US systems were equipped with a high-frequency linear probe (5-14 MHz). Three orthogonal diameters-the longest diameter and two perpendicular diameters-were measured, and the volume of each nodule was calculated using the following equation: $V=\pi a b c / 6$ (where $\mathrm{V}$ is the volume, $\mathrm{a}$ is the longest diameter, and $\mathrm{b}$ and $\mathrm{c}$ are the two perpendicular diameters). The composition of each nodule was graded into four categories based on the proportion of the solid component: 1 , solid (no obvious cystic content); 2 , predominantly solid (cystic content $\leq 50 \%$ ); 3 , predominantly cystic (cystic content $>50 \%$ ); and 4, cystic (no obvious solid content).

Before the treatment, the physicians recorded a cosmetic score as follows: 1, no palpable mass; 2 , no cosmetic problem but a palpable mass; 3, a cosmetic problem on swallowing only; and 4, a readily and always detected cosmetic problem. Patient-determined symptom scores were rated on a visual analogue scale from 0 to 10 as baseline data. Benign cytological results were confirmed by experienced cytopathologists according to the Bethesda classification system.

\section{Assessment of QoL}

Thyroid-specific symptoms and QoL in patients with BSTNs were evaluated using a thyroid-specific QoL questionnaire, which was translated into Korean and validated for patients with thyroid disease (Supplementary Table 1). Considering that patients with BSTNs often face unique QoL problems as compared with those other types of neck masses, assessment using a disease-specific QoL questionnaire was considered important.

The questionnaire evaluated the physical, psychological, and spiritual dimensions, with responses scored on a scale from 0 (none) to 4 (very severe). The QoL questionnaire consisted of 11 separate scales in the following aspects: goiter symptoms (11 items), tiredness (7 items), cognition (6 items), anxiety (6 items), depression ( 7 items), emotional susceptibility ( 7 items), impaired social life (4 items), impaired daily life (6 items), impaired sex life (2 items), cosmetic concern (6 items), and overall QoL (1 item). Since this study only included patients with normal thyroid function, the scales evaluating symptoms of hyperthyroidism and hypothyroidism were excluded. The QoL questionnaire was administered to patients before the procedure and 1, 6, and 12 months after treatment. Two clinical research coordinators administered the interviews and questionnaires.

\section{Ablation Procedures}

All procedures were performed by two experienced radiologists who had evaluated the preprocedural US images (J.H.B. and E.J.H. with 24 and 14 years of clinical experience, respectively, in evaluating neck US images and performing the procedure).

RFA was performed using an 18-gauge monopolar modified internally cooled electrode (VIVA, STARmed) with a $0.5,0.7$, or 1.0 $\mathrm{cm}$ active tip, as well as a radiofrequency (RF) generator (VIVA RF generator, STARmed) and a peristaltic pump (VIVA pump, STARmed). Under US guidance, the ablation needle was inserted into the nodule by the trans-isthmic approach, and ablation was performed using the moving-shot technique, with 30-70 W of RF power, depending on the sizes of the treated nodule and active tip. Ablation was terminated when the entire target lesion was covered with hyperechoic microbubbles.

EA was performed by inserting an 18-gauge needle via the normal thyroid tissue to minimize leakage of ethanol outside the cyst. After aspiration of cystic fluid, $99 \%$ sterile ethanol was injected through the same needle. The amount of injected ethanol was less than $50 \%$ of the aspirated fluid volume. After 5-10 minutes with the needle in place, the injected ethanol was removed completely, and the needle was withdrawn.

Procedure-related pain was graded into four categories and recorded. In RF procedures, the four categories were as follows: grade 0 , RF power did not have to be turned off because the patient experienced no pain; grade 1, RF power was turned off once or twice to reduce pain levels; grade 2, RF power was turned off more than three times; and grade 3, RF procedure was incompletely terminated due to severe pain. In EA procedures, the four categories were as follows: grade 0 , no pain or mild pain similar to the pain experienced during the lidocaine injection; grade 1, pain greater than the lidocaine injection, but not needing medication; grade 2, pain needing medication; and grade 3, EA procedure terminated before completion due to severe pain. Complications and side effects were defined according to the quality improvement guidelines of the Society of Interventional Radiology and a prior multicenter evaluation of complications. Each patient was monitored in the hospital for 2 hours after RFA and for 30 minutes after EA.

\section{Follow-up and Outcome Assessment}

US examinations were performed at 1,6, and 12 months after RFA or EA. In the US examinations, changes in nodule size and volume were evaluated. The QoL questionnaire was administered during each visit, and improvements in patients' symptoms and cosmetic problems were evaluated. Any adverse events occurring during the 12-month follow-up period were recorded.

\section{Statistical Analysis}

Categorical variables are presented as numbers and percentages, 
and continuous variables are expressed as mean \pm standard deviation. Linear mixed-effects modeling was performed for longitudinal data analysis. The model included group, time, and an interaction between group and time as the fixed effects. Patterns of covariance among repeated observations were modeled to account for correlations among observations for each subject, with covariance patterns selected from comparisons between models using the likelihood ratio test. The model parameter was obtained using the residual maximum likelihood estimation method, implemented with a Newton-Raphson algorithm. All P-values were two-sided, and a P-value $<0.05$ was considered to indicate statistically significant. Statistical analyses were performed using SPSS for Windows version 23.0 (IBM Corp., Armonk, NY, USA).

\section{Results}

\section{Patient Demographics and Clinical Characteristics}

The baseline clinical and treatment characteristics of the patients are summarized in Table 1. The mean longest size and mean volume of the index nodule were $4.2 \pm 1.5 \mathrm{~cm}$ and $21.6 \pm 22.1 \mathrm{~mL}$, respectively. They showed no significant differences between the EA and RFA groups in the longest mean nodule size $(4.1 \mathrm{~cm}$ vs. $4.3 \mathrm{~cm}, \mathrm{P}=0.526)$ and mean nodule volume $(22.0 \mathrm{~mL}$ vs. $21.3 \mathrm{~mL}$, $\mathrm{P}=0.248)$. Of the $31 \mathrm{BSTN}$ s that underwent $E A, 23(74.2 \%)$ were predominantly cystic and eight $(25.8 \%)$ were cystic, and of the 55 BSTNs which underwent RFA, 30 (54.5\%) were predominantly solid and 25 (45.5\%) were solid. Of the 86 patients, 77 (89.5\%) underwent one session of treatment and nine $(10.5 \%$, one EA case and eight RFA cases) underwent two sessions. The mean procedurerelated pain was $1.0 \pm 0.7$ during RFA and $0.0 \pm 0.2$ during EA.

\section{Treatment Efficacy}

Table 2 shows changes in the size and volume of BSTNs over time after treatment. After the procedures, significant reductions in the mean volume of the ablated nodules were noted at 1-, 6-, and 12-month follow-ups (all $\mathrm{P}<0.001$ ). In comparison between the EA group and RFA group, the mean VRR of the EA group was significantly higher than that of the RFA group at the $1-, 6-$, and 12-month follow-ups $(78.7 \% \pm 16.1 \%$ vs. $49.1 \% \pm 15.8 \%, P<0.001$; $86.3 \% \pm 21.7 \%$ vs. $73.0 \% \pm 14.5 \%, P=0.002$; and $90.9 \% \pm 14.9 \%$ vs. $80.3 \% \pm 12.4 \%, \mathrm{P}=0.004$, respectively).

After the procedures, both symptom and cosmetic scores

Table 1. Baseline characteristics of patients with benign thyroid nodules included in this study

\begin{tabular}{lc}
\hline \multicolumn{1}{c}{ Characteristic $(\mathrm{n}=86)$} & Value \\
\hline Age (year) & $54.9 \pm 12.9(24-83)$ \\
Sex & $18(20.9)$ \\
Male & $68(79.1)$ \\
Female & $10.1 \pm 4.8$ \\
Follow-up duration (month) & \\
No. of treatment sessions & $77(89.5)$ \\
One & $9(10.5)$ \\
Two & \\
Nodule composition & $25(29.1)$ \\
Solid & $31(36.0)$ \\
Predominantly solid & $22(25.6)$ \\
Predominantly cystic & $8(9.3)$ \\
Cystic & $4.2 \pm 1.5(1.3-8.2)$ \\
Longest nodule diameter (cm) & $21.6 \pm 22.1(0.5-118.1)$ \\
Nodule volume (mL)
\end{tabular}

Values are presented as mean \pm standard deviation (range) or number (\%).

Table 2. Ultrasound features and cosmetic and symptom scores in patients before and after the procedures

\begin{tabular}{|c|c|c|c|c|c|c|c|c|c|c|c|c|}
\hline & \multicolumn{4}{|c|}{ Total $(n=86)$} & \multicolumn{4}{|c|}{ Ethanol ablation $(n=31)$} & \multicolumn{4}{|c|}{ Radiofrequency ablation $(n=55)$} \\
\hline & Baseline & $\begin{array}{c}1 \mathrm{mo} \\
(\mathrm{n}=85)\end{array}$ & $\begin{array}{c}6 \mathrm{mo} \\
(\mathrm{n}=67)\end{array}$ & $\begin{array}{l}12 \mathrm{mo} \\
(\mathrm{n}=60)\end{array}$ & Baseline & $\begin{array}{c}1 \mathrm{mo} \\
(\mathrm{n}=30)\end{array}$ & $\begin{array}{c}6 \mathrm{mo} \\
(\mathrm{n}=19)\end{array}$ & $\begin{array}{l}12 \mathrm{mo} \\
(\mathrm{n}=16)\end{array}$ & Baseline & $\begin{array}{c}1 \mathrm{mo} \\
(\mathrm{n}=55)\end{array}$ & $\begin{array}{c}6 \mathrm{mo} \\
(\mathrm{n}=48)\end{array}$ & $\begin{array}{l}12 \mathrm{mo} \\
(\mathrm{n}=44)\end{array}$ \\
\hline $\begin{array}{l}\text { Longest tumor } \\
\text { diameter }(\mathrm{cm})\end{array}$ & $4.2 \pm 1.5$ & $3.1 \pm 1.3$ & $2.5 \pm 1.3$ & $2.1 \pm 1.2$ & $4.1 \pm 1.6$ & $2.5 \pm 1.3$ & $1.9 \pm 1.1$ & $1.1 \pm 0.8$ & $4.3 \pm 1.4$ & $3.5 \pm 1.3$ & $2.7 \pm 1.3$ & $2.4 \pm 1.2$ \\
\hline Tumor volume (mL) & $21.6 \pm 22.0$ & $8.6 \pm 10.4$ & $6.0 \pm 9.4$ & $4.0 \pm 6.1$ & $22.0 \pm 24.8$ & $5.3 \pm 7.9$ & $2.9 \pm 4.3$ & $0.9 \pm 1.7$ & $21.3 \pm 20.7$ & $10.5 \pm 11.2$ & $7.3 \pm 10.6$ & $5.1 \pm 6.8$ \\
\hline $\begin{array}{l}\text { Volume reduction } \\
\text { ratio (\%) }\end{array}$ & - & $59.6 \pm 21.1$ & $76.7 \pm 17.6$ & $83.1 \pm 13.7$ & - & $78.7 \pm 16.1$ & $86.3 \pm 21.7$ & $90.9 \pm 14.9$ & - & $49.1 \pm 15.8$ & $73.0 \pm 14.5$ & $80.3 \pm 12.4$ \\
\hline Cosmetic score $(1-4)$ & $3.8 \pm 0.6$ & $2.8 \pm 1.0$ & $2.2 \pm 1.0$ & $1.9 \pm 0.9$ & $3.9 \pm 0.4$ & $2.4 \pm 1.1$ & $1.8 \pm 0.9$ & $1.8 \pm 1.2$ & $3.7 \pm 0.6$ & $3.1 \pm 0.9$ & $2.4 \pm 1.0$ & $1.9 \pm 0.8$ \\
\hline $\begin{array}{l}\text { Symptom score } \\
(0-10)\end{array}$ & $3.5 \pm 1.9$ & $1.2 \pm 1.0$ & $0.8 \pm 0.8$ & $0.6 \pm 0.7$ & $3.7 \pm 2.2$ & $1.0 \pm 0.8$ & $0.4 \pm 0.5$ & $0.4 \pm 0.7$ & $3.4 \pm 1.8$ & $1.3 \pm 1.1$ & $1.0 \pm 0.8$ & $0.7 \pm 0.7$ \\
\hline $\begin{array}{l}\text { Pain during the } \\
\text { procedure }(0-3)\end{array}$ & $0.8 \pm 0.7$ & - & - & - & $0.0 \pm 0.2$ & - & - & - & $1.0 \pm 0.7$ & - & - & - \\
\hline
\end{tabular}

Values are presented as mean \pm standard deviation. 
decreased significantly at the $1-, 6-$, and 12 -month follow-ups (all $\mathrm{P}<0.001$ ). The symptom scores were not significantly different between the EA group and the RFA group at any time point after the procedures. However, the cosmetic scores were significantly lower in the EA group than in the RFA group at the 1-and 6-month follow-ups ( $2.4 \pm 1.1$ vs. $3.1 \pm 0.9, P=0.002$ and $1.8 \pm 0.9$ vs. $2.4 \pm 1.0$, $\mathrm{P}=0.009$, respectively); while there were no significant differences at the 12-month follow-up (1.8 \pm 1.2 vs. $1.9 \pm 0.8, P=0.443)$.

\section{QoL at Baseline and Follow-up}

Table 3 shows the thyroid-specific QoL scores in these patients at baseline and 1, 6, and 12 months after treatment. The scores on each scale of the QoL questionnaire showed a significant improvement from the baseline to 1,6 , and 12 months after RFA or EA (all $P<0.001$ ). The overall QoL, which was scored from 0 (good) to 4 (poor), persistently improved from baseline to the 12-month follow-up over time (from $1.7 \pm 0.9$ to $0.6 \pm 0.7$ ). Fig. 2 shows the score improvement percentage for each scale. The scores for anxiety and cosmetic concerns showed an improvement of more than $80 \%(83.2 \%$ and $84.9 \%$, respectively). In contrast, the scores of cognition, tiredness, and depression showed an improvement of about $50 \%$ (51.7\%, 54.6\%, and $61.1 \%$, respectively).

Table 3. Thyroid-specific quality-of-life scores before and after the procedures

\begin{tabular}{|c|c|c|c|c|c|c|c|c|c|c|c|c|}
\hline \multirow{2}{*}{ Scales ${ }^{a)}$} & \multicolumn{4}{|c|}{ Total } & \multicolumn{4}{|c|}{ Ethanol ablation } & \multicolumn{4}{|c|}{ Radiofrequency ablation } \\
\hline & Baseline & $1 \mathrm{mo}$ & $6 \mathrm{mo}$ & $12 \mathrm{mo}$ & aseline & $1 \mathrm{mo}$ & $6 \mathrm{mo}$ & $12 \mathrm{mo}$ & Baseline & $1 \mathrm{mo}$ & $6 \mathrm{mo}$ & $12 \mathrm{mo}$ \\
\hline Goiter sympto & $11.5 \pm 8.7$ & $5.0 \pm 6.4$ & $4.5 \pm 7.0$ & $2 \pm 3.1$ & $2.6 \pm 9.0$ & $2.4 \pm 3.1$ & $4.0 \pm 9.0$ & $3.3 \pm 3.4$ & $10.9 \pm 8.6$ & $6.5 \pm 7.2$ & $4.8 \pm 6.1$ & $3.1 \pm 3.0$ \\
\hline Tiredness (7) & $10.8 \pm 5.7$ & $.5 \pm 4.4$ & $4 \pm 4.4$ & $9 \pm 3.4$ & $0.9 \pm 6.4$ & $6.2 \pm 4.5$ & $6.0 \pm 5.5$ & $0 \pm 3.0$ & $10.7 \pm 5.3$ & $8.2 \pm 4.3$ & $6.6 \pm 3.9$ & $5.2 \pm 3.5$ \\
\hline Cognition (6) & $.0 \pm 5.7$ & $4.1 \pm 4.5$ & $3.8 \pm 3.7$ & $2.9 \pm 3.5$ & $5.9 \pm 5.6$ & $4.5 \pm 5.8$ & $3.9 \pm 4.1$ & $3.2 \pm 4.3$ & $6.0 \pm 5.7$ & $3.9 \pm 3.6$ & $3.8 \pm 3.6$ & $2.8 \pm 3.2$ \\
\hline Anxiety (6) & $9.5 \pm 6.7$ & $3.6 \pm 4.3$ & $2.6 \pm 3.8$ & $1.6 \pm 3.0$ & $10.6 \pm 7.2$ & $3.4 \pm 4.1$ & $2.1 \pm 3.0$ & $1.5 \pm 3.3$ & $8.8 \pm 6.3$ & $3.7 \pm 4.4$ & $2.8 \pm 4.1$ & $1.7 \pm 3.0$ \\
\hline Depression (7) & $.0 \pm 6.5$ & $5.4 \pm 5.0$ & $4.2 \pm 3.9$ & & $10.1 \pm 7.1$ & $5.7 \pm 6.2$ & & & & & & $3.4 \pm 3.5$ \\
\hline Emotional susceptibility (7) & $7.4 \pm 7.0$ & & $3.4 \pm 4.6$ & & & & & & & & & \\
\hline Impaired social life (4) & $2.1 \pm 2.9$ & $1.2 \pm 2.3$ & $0.8 \pm 1.7$ & $0.7 \pm 1.6$ & $2.2 \pm 3.4$ & $1.1 \pm 2.4$ & $0.6 \pm 0.9$ & $0.9 \pm 1.7$ & $2.0 \pm 2.7$ & $1.3 \pm 2.3$ & $0.9 \pm 2.0$ & $0.6 \pm 1.5$ \\
\hline Impaired daily life (6) & $3.9 \pm 5.1$ & $1.6 \pm 2.9$ & $1.5 \pm 3.7$ & $0.8 \pm 1.8$ & $3.8 \pm 4.9$ & $1.0 \pm 2.2$ & $1.9 \pm 5.2$ & $0.8 \pm 1.5$ & $4.0 \pm 5.3$ & $1.9 \pm 3.3$ & $1.3 \pm$ & $0.8 \pm 1.9$ \\
\hline Impaired sex life (2) & $0.8 \pm 1.6$ & $0.6 \pm 1.3$ & $0.6 \pm 1.3$ & $0.3 \pm 1.0$ & $0.4 \pm 0.9$ & $0.3 \pm 0.8$ & $0.4 \pm 1.0$ & $0.1 \pm 0.3$ & $1.0 \pm 1.9$ & $0.8 \pm 1.5$ & $0.7 \pm 1.4$ & $0.4 \pm 1.1$ \\
\hline Cosmetic concern (6) & $5.3 \pm 6.0$ & $2.0 \pm 3.2$ & $1.6 \pm 3.5$ & $0.8 \pm 1.7$ & $7.7 \pm 6.9$ & $1.7 \pm 3.4$ & $1.7 \pm 5.2$ & $1.1 \pm 2.7$ & $3.9 \pm 4.9$ & $2.2 \pm 3.0$ & $1.6 \pm 2.5$ & $0.7 \pm 1.2$ \\
\hline Overall quality of life & $1.7 \pm 0.9$ & $1.2 \pm 0.9$ & $0.9 \pm 0.9$ & $0.6 \pm 0.7$ & $1.7 \pm 0.7$ & $1.3 \pm 1.0$ & $0.7 \pm 0.7$ & $0.8 \pm 0.7$ & $1.6 \pm 1.1$ & $1.2 \pm 0.8$ & $1.0 \pm 0.9$ & $0.5 \pm 0.7$ \\
\hline
\end{tabular}

All results are reported as mean \pm standard deviation, with all scores differing significantly from baseline ( $<<0.001$ each).

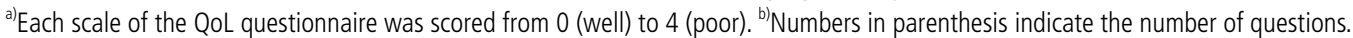

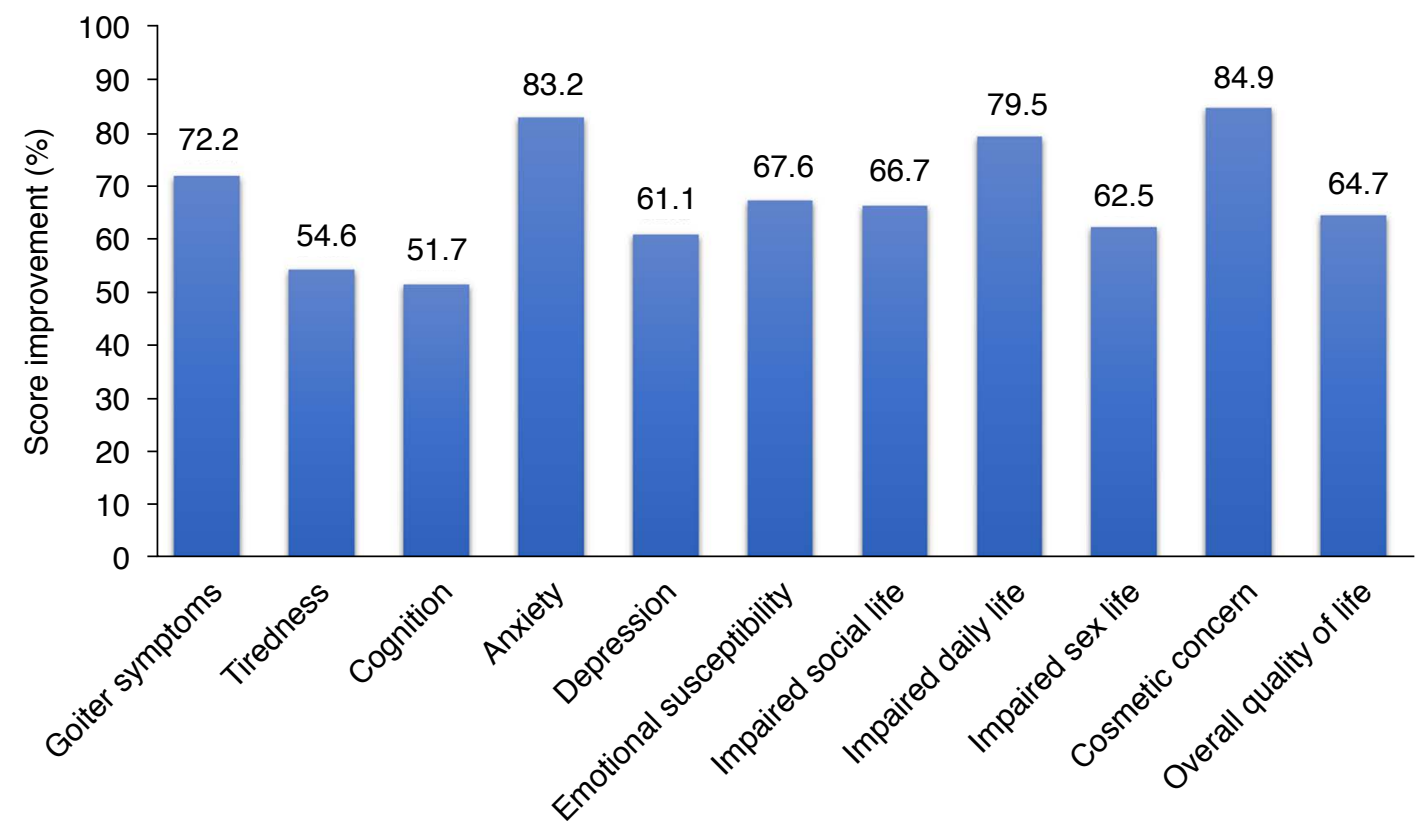

Fig. 2. Score improvement percentage of 11 scales for thyroid-specific quality of life. 
Before the procedures, cosmetic concern scores were significantly higher in the EA group than in the RFA group ( $7.7 \pm 6.9$ vs. $3.9 \pm 4.9$, $\mathrm{P}=0.002$ ), but there were no statistically significant between-group differences at the 1-, 6-, and 12-month follow-ups. Goiter symptom scores decreased more rapidly in the EA group than those in the RFA group at 1 month ( $2.4 \pm 3.1$ vs. $6.5 \pm 7.2, P=0.002$ ), but there were no significant differences at 6 and 12 months. Scores for tiredness, cognition, anxiety, depression, emotional susceptibility, impaired social/daily/sex life, and overall QoL during the follow-up period were not significantly different between the two groups (all $P>0.05)$.

According to the baseline nodule volume, the patients were divided into two groups (small to medium, $<20 \mathrm{~mL}$; and large, $\geq 20$ $\mathrm{mL}$ ), and analyzed the anxiety scores between the groups. Patients with a baseline nodule volume larger than $20 \mathrm{~mL}$ had higher anxiety scores than patients with a baseline nodule volume less than $10 \mathrm{~mL}$ $(8.8 \pm 6.3$ vs. $10.4 \pm 7.1, P=0.295)$. However, this tendency did not have clinical significance.

\section{Safety}

Several patients who underwent RFA and EA reported mild pain at the treated sites, or pain radiating to the head, ear, shoulder, or teeth during the procedure. However, the pain was reduced when the generator output was reduced or turned off during ablation. All patients tolerated RFA or EA. There were no major complications, such as nerve injury or nodule rupture.

\section{Discussion}

This study prospectively evaluated changes in thyroid-specific QoL scores after RFA or EA in patients with BSTNs. Both procedures significantly improved all thyroid-specific QoL scales (all $P<0.001$ ) and reduced mean nodule volume by $80.3 \%-90.9 \%$ at the 12-month follow-up.

US-guided minimally invasive treatments, such as RFA or EA, have been proposed for the management of patients with BSTNs during the past two decades $[1,2]$. Current international practice guidelines generally recommend RFA for nodules with a solid component $\geq 50 \%$ and EA for nodules with a solid component $<50 \%[2,16$ 18]. However, it is not well known whether the effects of these treatments eventually improve QoL in these patients. In this study, thyroid-specific QoL, including all the physical, psychological, and spiritual dimensions, significantly improved after RFA or EA. Goiter symptoms resolved more rapidly in the EA group than those in the RFA group at the 1-month follow-up (2.4 \pm 3.1 vs. $6.5 \pm 7.2)$, but there were no differences at the last follow-up $(3.3 \pm 3.4$ vs. $3.1 \pm 7.2)$. Other symptom scales also improved persistently in both groups. By contrast, a previous study by Oddo et al. [13] reported no significant improvement in QoL scales after RFA, except for the "general score," which improved after 3 months. They explained that the cause of less improvement seemed to have been due to the low VRR, which was $<50 \%$ in most patients. In comparison, in the present study, it was possible to achieve a much higher VRR at the 12 -month follow-up $(90.9 \% \pm 14.9 \%$ in the EA group and $80.3 \% \pm 12.4 \%$ in the RFA group) and all thyroid-specific QoL scales in both groups improved after treatment. Sufficient VRR should be achieved by trained operators using a unified protocol and similar devices to improve thyroid-specific QoL after the procedure.

The scores for items that were directly related to nodule size such as anxiety and cosmetic concern showed a greater improvement $(83.2 \%$ and $84.9 \%$, respectively) than the others. Moreover, patients with larger baseline nodule volume tended to have higher anxiety scores ( $8.8 \pm 6.3$ vs. $10.4 \pm 7.1, P=0.295)$. However, the scores for items that were relatively uncorrelated with nodule size such as cognition, tiredness, and depression showed relatively less improvement $(51.7 \%, 54.6 \%$, and $61.1 \%$, respectively). This study did not include patients with functioning nodules, and it appears that the items that showed relatively less improvement were related to thyroid function rather than nodule size. The inclusion of patients with functioning nodules might change such results.

Surgery is a long-established therapeutic option for BSTNs $[19,20]$. However, the cost of thyroid surgery, the risk of temporary or permanent complications, and the effect on QoL remain relevant concerns [12,21]. Regarding QoL after surgery, several previous studies demonstrated that surgery may not lead to an overall improvement in QoL in patients with BSTNs [22,23]. In a prospective observational study by a German group of surgeons [23], QoL scores did not change in most patients after surgery, while $21 \%-30 \%$ showed improvements and 14\% showed a deterioration in QoL. Regarding these issues, Yue et al. [12] compared the QoL scores of propensity score-matched patients with benign thyroid nodules that were treated with RFA or surgery. In their study, patients treated with RFA had significantly better QoL than patients treated with surgery for general health, vitality, and mental health at a 6-month follow-up, while the QoL scores were significantly improved on roleemotional and mental health in both groups. Further studies of direct comparison with surgery are necessary for these issues.

QoL is one of the key considerations when deciding the clinical management for patients with thyroid lesions [24]. US-guided minimally invasive treatments, such as RFA or EA, are also promising for QoL improvement in patients with thyroid cancer. In a recent prospective study by Morris-Wiseman [25], thyroid cancer patients with postoperative permanent hypoparathyroidism had significantly lower QoL, with nearly three to five times as many general symptom- 
related problems, as patients without hypoparathyroidism. Several studies have reported that RFA showed good clinical outcomes, with better QoL, compared with surgery in patients with recurrent thyroid cancers in the dissected neck [26-28]. Chung et al. [27] also demonstrated that RFA is effective for local tumor control, even for tumors invading the airways. In addition, several recent studies have shown that RFA is highly encouraging in terms of low morbidity and recurrence rates and high thyroid-cancer-specific QoL scores in patients with low-risk papillary thyroid microcarcinoma [26]. Although all treatments have potential risks, clinicians managing thyroid nodules should have a multitude of sources to consult for optimal personalized management. Patients should be fully informed regarding QoL before selecting a treatment option.

This study had several limitations. Although a VRR of more than $80 \%$ was achieved after EA or RFA within 12 months, longterm follow-up results are necessary to evaluate the actual clinical impact of these ablation treatments on QoL. In addition, this study did not include patients with functioning nodules. Because proper management of functioning nodules remains controversial, further studies are necessary to achieve better outcomes with fewer side effects.

In conclusion, both RFA and EA are safe and effective in thyroidspecific QoL as well as improving the reduction of nodule volume in patients with BSTNs.

ORCID: So Yeong Jeong: https://orcid.org/0000-0003-4705-0008; Eun Ju Ha: https:// orcid.org/0000-0002-1234-2919; Jung Hwan Baek: https://orcid.org/0000-00030480-4754; Tae Yong Kim: https://orcid.org/0000-0003-4982-4441; Yu-Mi Lee: https://orcid.org/0000-0002-8183-2604; Jeong Hyun Lee: https://orcid.org/00000002-0021-4477; Jeonghun Lee: https://orcid.org/0000-0003-4925-9104

\section{Author Contributions}

Conceptualization: Jeong SY, Ha EJ, Baek JH. Data acquisition: Jeong SY, Ha EJ, Baek JH, Lee YM. Data analysis or interpretation: Jeong SY, Ha EJ, Baek JH, Kim TY, Lee JH, Lee J. Drafting of the manuscript: Jeong SY, Baek JH. Critical revision of the manuscript: Ha EJ, Baek $J H$, Kim TY, Lee YM, Lee JH, Lee J. Approval of the final version of the manuscript: all authors.

\section{Conflict of Interest}

No potential conflict of interest relevant to this article was reported.

\section{Supplementary Material}

Supplementary Table 1. Thyroid-specific QoL questionnaire (https:// doi.org/10.14366/usg.21003).

\section{References}

1. Hahn SY, Shin JH, Na DG, Ha EJ, Ahn HS, Lim HK, et al. Ethanol ablation of the thyroid nodules: 2018 consensus statement by the Korean Society of Thyroid Radiology. Korean J Radiol 2019;20:609620.

2. Kim JH, Baek JH, Lim HK, Ahn HS, Baek SM, Choi YJ, et al. 2017 Thyroid radiofrequency ablation guideline: Korean Society of Thyroid Radiology. Korean J Radiol 2018;19:632-655.

3. Baek JH, Ha EJ, Choi YJ, Sung JY, Kim JK, Shong YK. Radiofrequency versus ethanol ablation for treating predominantly cystic thyroid nodules: a randomized clinical trial. Korean J Radiol 2015;16:13321340.

4. Lee M, Baek JH, Suh CH, Chung SR, Choi YJ, Lee JH, et al. Clinical practice guidelines for radiofrequency ablation of benign thyroid nodules: a systematic review. Ultrasonography 2021;40:256-264.

5. Lee MK, Baek JH, Chung SR, Choi YJ, Lee YM, Kim TY, et al. Effectiveness of injecting cold $5 \%$ dextrose into patients with nerve damage symptoms during thyroid radiofrequency ablation. Endocrinol Metab (Seoul) 2020;35:407-415.

6. Ahn HS, Kim SJ, Park SH, Seo M. Radiofrequency ablation of benign thyroid nodules: evaluation of the treatment efficacy using ultrasonography. Ultrasonography 2016;35:244-252.

7. Cho SJ, Baek JH, Chung SR, Choi YJ, Lee JH. Long-term results of thermal ablation of benign thyroid nodules: a systematic review and meta-analysis. Endocrinol Metab (Seoul) 2020;35:339-350.

8. Chen $F$, Tian $G$, Kong $D$, Zhong $L$, Jiang $T$. Radiofrequency ablation for treatment of benign thyroid nodules: a PRISMA-compliant systematic review and meta-analysis of outcomes. Medicine (Baltimore) 2016;95:e4659.

9. Jung SL, Baek JH, Lee JH, Shong YK, Sung JY, Kim KS, et al. Efficacy and safety of radiofrequency ablation for benign thyroid nodules: a prospective multicenter study. Korean J Radiol 2018;19:167-174.

10. Valcavi $R$, Tsamatropoulos P. Health-related quality of life after percutaneous radiofrequency ablation of cold, solid, benign thyroid nodules: a 2-year follow-up study in 40 patients. Endocr Pract 2015;21:887-896.

11. Oddo S, Felix E, Mussap M, Giusti M. Quality of life in patients treated with percutaneous laser ablation for non-functioning benign thyroid nodules: a prospective single-center study. Korean J Radiol 2018;19:175-184.

12. Yue WW, Wang $S R$, Li XL, Xu HX, Lu F, Sun $L P$, et al. Quality of life and cost-effectiveness of radiofrequency ablation versus open surgery for benign thyroid nodules: a retrospective cohort study. Sci Rep 2016;6:37838.

13. Oddo S, Felix E, Repetto AM, Mussap M, Giusti M. Quality of life in patients treated with radiofrequency ablation for thyroid nodules. Res J Endocrinol Metab 2016:4:1.

14. Chung MS, Choi YJ, Kim SO, Lee YS, Hong JY, Lee JH, et al. A 
scoring system for prediction of cervical lymph node metastasis in patients with head and neck squamous cell carcinoma. AJNR Am J Neuroradiol 2019;40:1049-1054.

15. Son HM, Kim JH, Kim SC, Yoo RE, Bae JM, Seo H, et al. Distribution and malignancy risk of six categories of the pathology reporting system for thyroid core-needle biopsy in 1,216 consecutive thyroid nodules. Ultrasonography 2020;39:159-165.

16. Papini E, Pacella CM, Solbiati LA, Achille G, Barbaro D, Bernardi $S$, et al. Minimally-invasive treatments for benign thyroid nodules: a Delphi-based consensus statement from the Italian minimallyinvasive treatments of the thyroid (MITT) group. Int J Hyperthermia 2019;36:376-382.

17. Garberoglio R, Aliberti C, Appetecchia M, Attard M, Boccuzzi G, Boraso F, et al. Radiofrequency ablation for thyroid nodules: which indications? The first Italian opinion statement. J Ultrasound 2015; 18:423-430.

18. Park HS, Yim Y, Baek JH, Choi YJ, Shong YK, Lee JH. Ethanol ablation as a treatment strategy for benign cystic thyroid nodules: a comparison of the ethanol retention and aspiration techniques. Ultrasonography 2019;38:166-171.

19. Haugen BR, Alexander EK, Bible KC, Doherty GM, Mandel SJ, Nikiforov YE, et al. 2015 American Thyroid Association management guidelines for adult patients with thyroid nodules and differentiated thyroid cancer: the American Thyroid Association Guidelines Task Force on Thyroid Nodules and Differentiated Thyroid Cancer. Thyroid 2016:26:1-133.

20. Gharib H, Papini E, Paschke R, Duick DS, Valcavi R, Hegedus L, et al. American Association of Clinical Endocrinologists, Associazione Medici Endocrinologi, and European Thyroid Association Medical guidelines for clinical practice for the diagnosis and management of thyroid nodules: executive summary of recommendations. Endocr Pract 2010;16:468-475.

21. Bergenfelz A, Jansson S, Kristoffersson A, Martensson $H$, Reihner $E$, Wallin $G$, et al. Complications to thyroid surgery: results as reported in a database from a multicenter audit comprising 3,660 patients. Langenbecks Arch Surg 2008;393:667-673.

22. Promberger R, Hermann M, Pallikunnel SJ, Seemann R, Meusel M, Ott J. Quality of life after thyroid surgery in women with benign euthyroid goiter: influencing factors including Hashimoto's thyroiditis. Am J Surg 2014;207:974-979.

23. Schmitz-Winnenthal FH, Schimmack S, Lawrence B, Maier U, Heidmann M, Buchler MW, et al. Quality of life is not influenced by the extent of surgery in patients with benign goiter. Langenbecks Arch Surg 2011;396:1157-1163.

24. Barbus E, Pestean C, Larg MI, Piciu D. Quality of life in thyroid cancer patients: a literature review. Clujul Med 2017;90:147-153.

25. Morris-Wiseman LF. Decreased quality of life in patients with postoperative hypoparathyroidism after thyroid cancer surgery. Clin Thyroidol 2020;32:534-536.

26. Zhang M, Tufano RP, Russell JO, Zhang Y, Zhang Y, Qiao Z, et al. Ultrasound-guided radiofrequency ablation versus surgery for low-risk papillary thyroid microcarcinoma: results of over 5 years follow-up. Thyroid 2020;30:408-417.

27. Chung SR, Baek JH, Choi YJ, Sung TY, Song DE, Kim TY, et al. Efficacy of radiofrequency ablation for recurrent thyroid cancer invading the airways. Eur Radiol 2021;31:2153-2160.

28. Yoo RE, Kim JH, Paeng JC, Park YJ. Radiofrequency ablation for treatment of locally recurrent thyroid cancer presenting as a metastatic lymph node with dense macrocalcification: a case report and literature review. Medicine (Baltimore) 2018;97:e0003. 